\title{
A-type stars: Evolution, rotation and binarity
}

\author{
Arlette Noels ${ }^{1}$, Josefina Montalbán ${ }^{1}$ and Carla Maceroni ${ }^{2}$ \\ ${ }^{1}$ Institut d'Astrophysique et Géophysique Université de Liège, \\ Allée du 6 Aôut, B-4000 Liège, Belgium \\ email: first.last@ulg.ac.be \\ ${ }^{2}$ INAF - Osservatorio Astronomico di Roma, \\ via Frascati 33, I-00040 Monteporzio C. (RM) - Italy \\ email: maceroni@coma.mporzio.astro.it
}

\begin{abstract}
We discuss the internal structure of stars in the mass range 1.5 to $4 M_{\odot}$ from the PMS to the subgiant phase with a particular emphasis on the convective core and the convective superficial layers. Different physical aspects are considered such as overshooting, treatment of convection, microscopic diffusion and rotation. Their influence on the internal structure and on the photospheric chemical abundances is briefly described.

The role of binarity in determining the observed properties and as a tool to constrain the internal structure is also introduced and the current limits of theories of orbital evolution and of available binary datasets are discussed.
\end{abstract}

Keywords. Stars: evolution, binaries: general, stars: rotation

\section{Introduction}

The theoretical evolution of A stars is extremely simple to compute if one ignores complex phenomena such as gravitational settling, radiative forces, rotation, turbulent mixing, magnetic fields, binarity... We shall briefly introduce the infuence of different treatments of convection, diffusion and rotation after a presentation of a "conservative" situation in which the only mixings come from convection and overshooting. Binarity as a tool to constrain stellar models is then discussed.

\section{A-type stars: evolution}

As A stars are located on or near the main sequence, we shall start this discussion with the core hydrogen burning phase.

\subsection{Core hydrogen burning}

Figure 1 (lp) shows the evolutionary track of a $3 M_{\odot}$ with a solar chemical composition.

The evolution with time of the radiative gradient, given by

$$
\nabla \mathrm{rad} \sim \frac{L}{m} \kappa
$$

is shown in Figure 2 (lp) in the inner $30 \%$ of the mass for selected models on the evolutionary path.

The adiabatic gradient, $\nabla_{\text {ad }}$, is also drawn on this Figure and its decrease towards the centre shows that the influence of the radiation pressure increases with time. Starting from the right of the Figure, the radiative gradient increases when reaching the CNO burning central layers, due to the increasing value of $L / m$, and hydrogen burning thus 

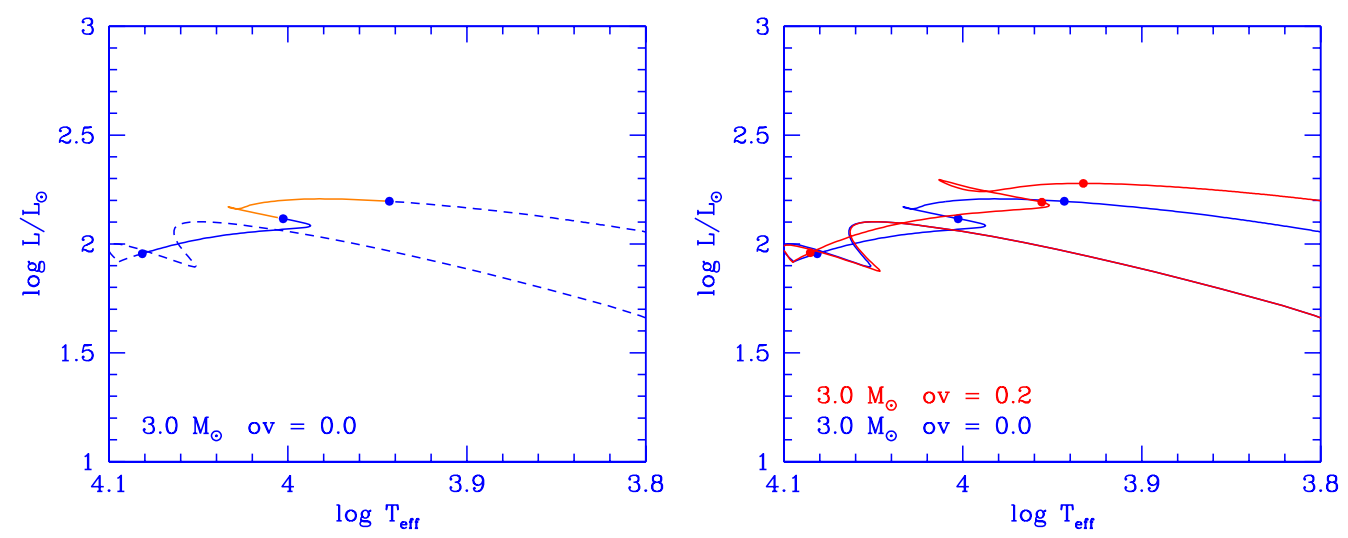

Figure 1. Left panel (lp): The evolutionary track for a $3 M_{\odot}$ star computed without overshooting. Right panel (rp): Same Figure with an overshooting parameter of 0.2 showing a wider MS track than in the lp.
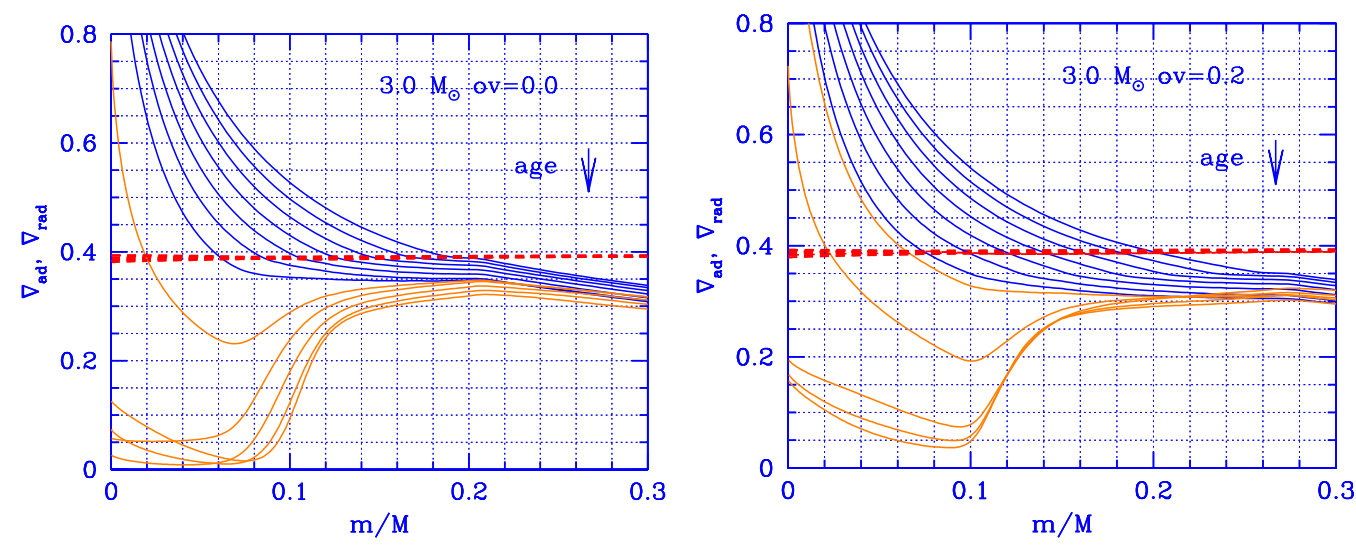

Figure 2. Left panel (lp): Radiative gradient for different models on the main sequence (black curves - first two dots starting from the left in Figure 1) and in the early $\mathrm{H}$ shell burning phase (gray curves - last two dots starting from the left in Figure 1). Right panel (rp): Same Figure with an overshooting parameter of 0.2 .

takes place in a convective core. Inside this core, the hydrogen abundance, $X$, decreases. The opacity, $\kappa$, being proportional to $(1+X)$, decreases as time goes on. This means that the convective core has its maximum extension in mass on the ZAMS and then slowly recedes as hydrogen is transformed into helium. The difference in $X$ between the core and the non-burning regions thus increases with time without any discontinuity at the edge since the convective core recedes. A gradient of hydrogen builds up and a plateau in $\nabla$ rad appears (see Figure $2(\mathrm{lp})$ ).

The decrease of $X$ leads to an increase in the temperature (Figure $3(\mathrm{lp})$ ) which is limited to the central layers while the other layers become cooler and cooler.

The central part of the star is contracting while the other layers are expanding. Once $X$ comes close to zero in the core, the increase in temperature becomes more pronounced (see Figure $3(\mathrm{lp})$ ). This is done by a global contraction (the so-called second gravitational contraction). 

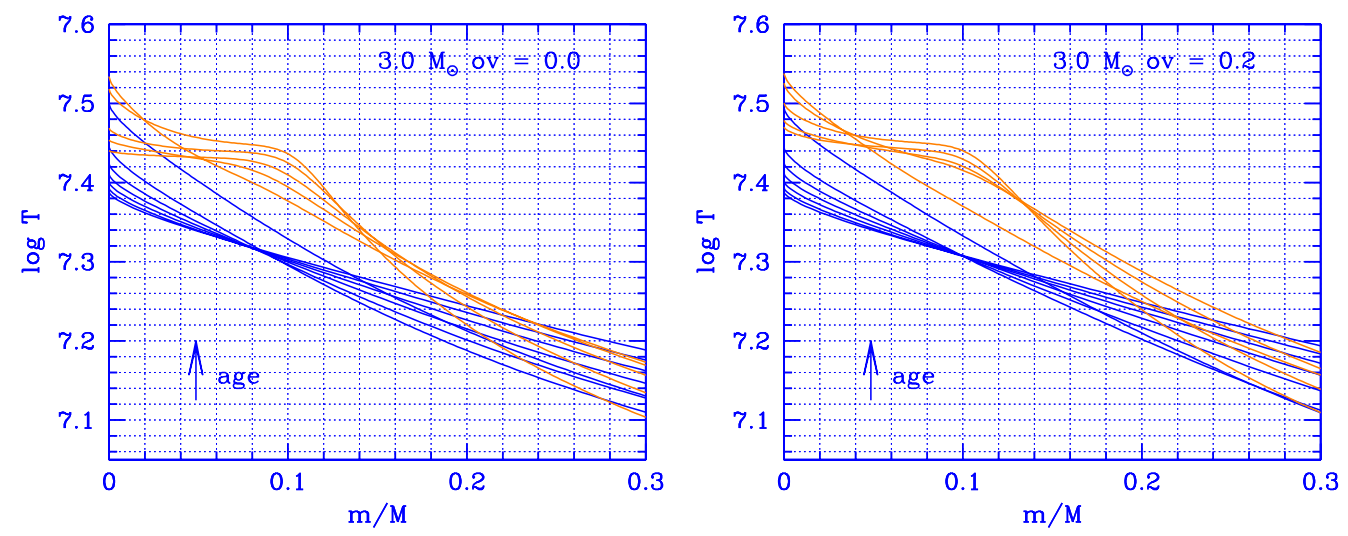

Figure 3. Left panel (lp): Temperature versus mass for different models on the main sequence (black curves - first two dots starting from the left in Figure 1) and in the early H shell burning phase (gray curves - last two dots starting from the left in Figure 1). Right panel (rp): Same Figure with an overshooting parameter of 0.2 .
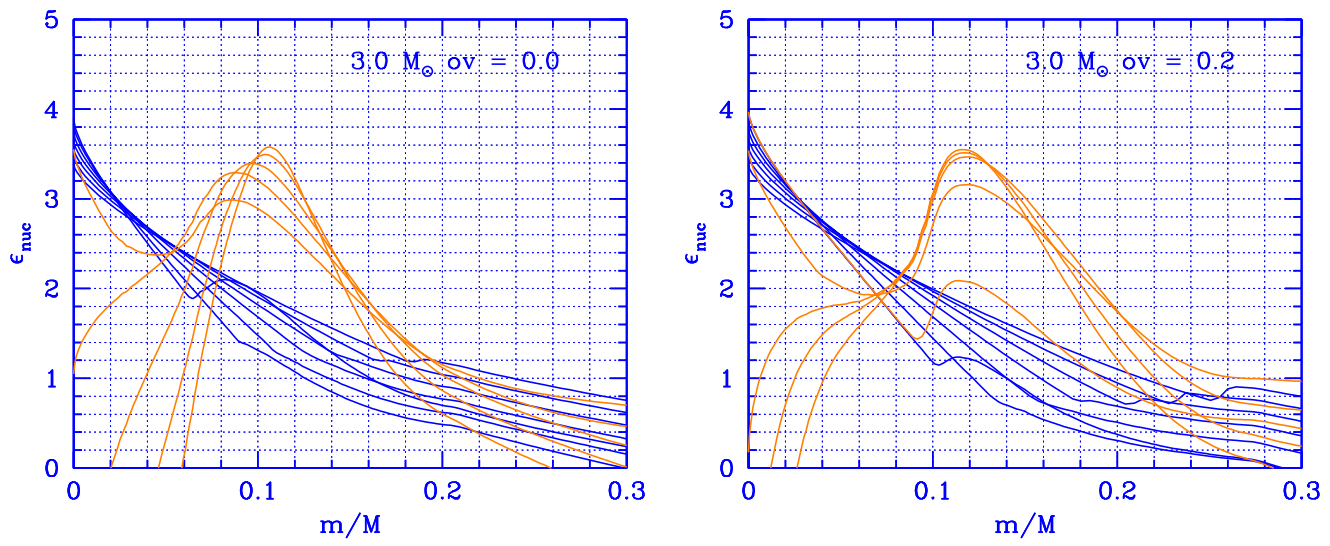

Figure 4. Left panel (lp): Nuclear energy rate versus mass for different models on the main sequence (black curves - first two dots starting from the left in Figure 1) and in the early $\mathrm{H}$ shell burning phase (gray curves - last two dots starting from the left in Figure 1). Right panel (rp): Same Figure with an overshooting parameter of 0.2 .

\subsection{Hydrogen shell burning}

In the $\mu$-gradient zone, a hydrogen burning shell starts to develop. A secondary maximum is seen in $\nabla$ rad (Figure $2(\mathrm{lp}))$ as well as in the nuclear enregy rate, $\epsilon$ (Figure 4 (lp)). Soon after, $\nabla$ rad becomes smaller than $\nabla$ ad and the convective core vanishes. As the energy production stops, an isothermal core appears. The models are now located between the last two dots on the evolutionary track in Figure 1 (lp). As hydrogen is burned in the shell, the isothermal core increases in mass. However, its mass cannot exceed the limiting mass of Schönberg-Chandrasekhar, given by

$$
\left(\frac{m}{M}\right)_{\mathrm{SC}} \simeq 0.37 \frac{\mu_{\mathrm{e}}}{\mu_{\mathrm{i}}}
$$

which is of the order of 0.08 . When this value is reached, the core must contract to create a temperature gradient (see Figure $3(\mathrm{lp})$ ) and this is accompanied by an expansion of the envelope where the temperature decreases. 

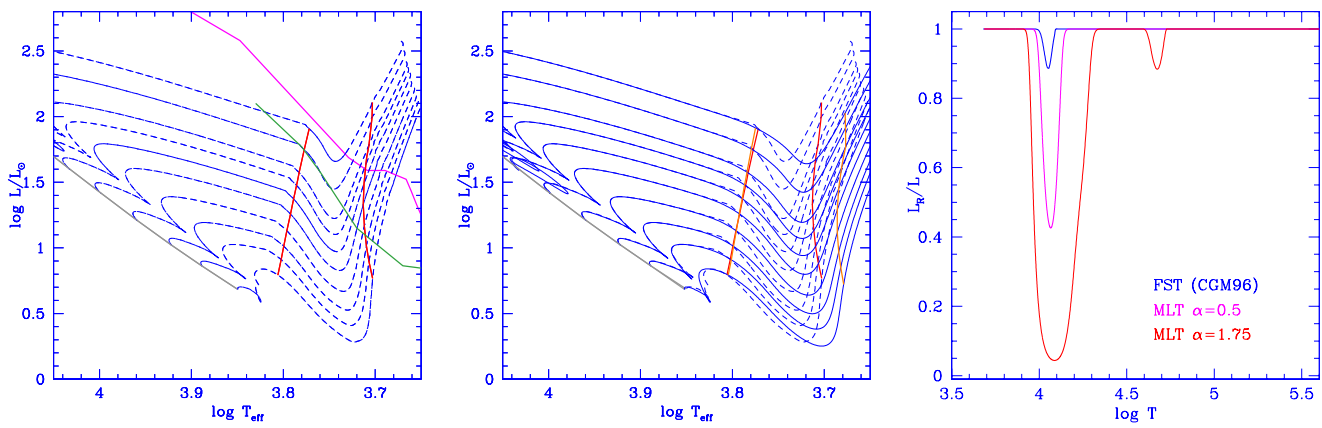

Figure 5. Left panel (lp): PMS tracks (see text) with two birthlines (bottom one: Palla \& Stahler (1993), top one: Behrend \& Maeder (2001). Center panel (cp): Evolutionary tracks computed with FST (dashed curves) and with MLT $(\alpha=1.6)$ (faded curves). Right panel (rp): The fraction of energy transported by radiation versus $\log T$ in the external layers. (1) With FST : Only one (the smallest) minimum near $\log T=4.1$ ( $\mathrm{H}$ I and $\mathrm{He} \mathrm{I}$ ) and no minimum near $\log T=4.7$. (2) With $M L T(\alpha=0.5)$ : One minimum (the intermediate one) near $\log T=4.1$ and no minimum near $\log T=4.7$. (3) With $M L T(\alpha=1.75)$ : One (the largest) minimum near $\log T=4.1$ and a second small one near $\log T=4.7$ (He II).

\subsection{Effect of overshooting}

To fit CM diagrams of open clusters as well as eclipsing binaries, overshooting seems to be needed with an extent increasing with stellar mass. We have computed a similar evolution with an overshooting parameter of 0.2 . A larger hydrogen reservoir due to the increase of the mixed region evidently translates into a longer core hydrogen burning phase as well as in a longer track in the HR diagram (Figure 1 (rp)). However, after the turn-off, for a similar variation in effective temperature, the evolution with overshooting is much more rapid. Figure 2 (rp) shows that $\nabla_{\text {rad }}$ never reaches zero so there is no plateau in temperature (Figure 3 (rp)). The reason lies in the fact that with the increase of the mixed region, the exhaustion of hydrogen takes place in a core whose mass is already greater than $\left(\frac{m}{M}\right)_{\mathrm{SC}}$. This results in a much quicker contraction of the central regions. The star moves more rapidly and for a similar change in effective temperature, the shell, shown by a maximum in the distribution of $\epsilon$, is still at the same mass fraction with overshooting while the shell has already moved significantly in mass without overshooting (compare lp with rp in Figure 4).

Although the $\mu$-gradient has a smaller slope with overshooting than without overshooting, the presence or absence of an isothermal core leads to a similar extent of the helium core at a similar effective temperature.

\subsection{Pre-main sequence evolution}

Let us now turn toward the pre-main sequence evolution (Figure 5). The two quasivertical lines on the Hayashi tracks and soon after show where the star becomes partly radiative and then completely radiative. The loops near the MS are the signatures of the CNO reactions evolving toward equilibrium. We have checked the effect of the new (smaller) cross-section of the slowest of the CNO reactions, i.e., ${ }^{14} \mathrm{~N}(p, \gamma){ }^{15} \mathrm{O}$ (Formicola \& LUNA 2004). Although the approach toward equilibrium is slower, the difference in the main sequence lifetime is not significant.

The points in the HR diagram where the stars become visible define the birthline. Two birthlines are drawn in Figure 5 (lp)).

The bottom one comes from Palla \& Stahler (1993) and is constructed assuming a constant accretion rate of $10^{-5} M_{\odot} /$ yr. This value was obtained by fitting the Herbig Ae 

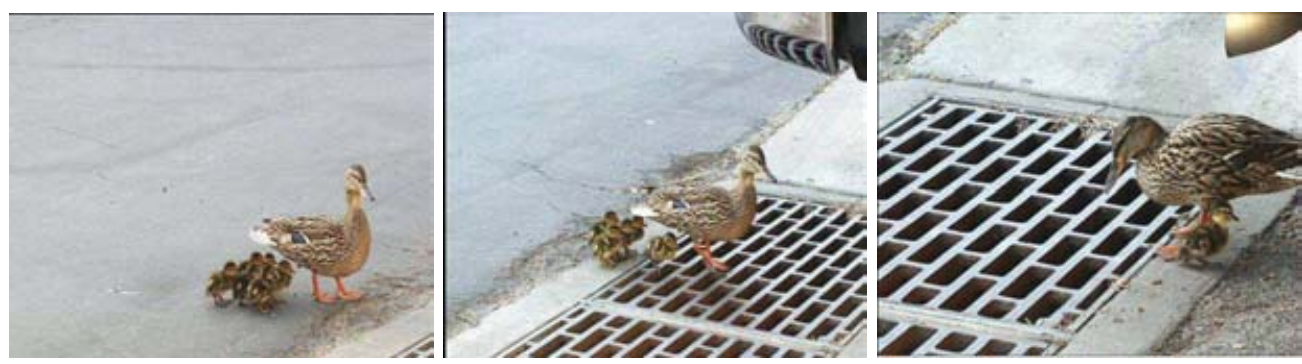

Figure 6. Illustration of diffusion. See text

and Be objects in the HR diagram. The top one comes from an analysis by Behrend \& Maeder (2001) where the accretion rate

$$
\dot{M}=\frac{1}{3} \dot{M}_{\text {disk }}
$$

has been derived to reproduce the observations in the HR diagram, in particular for the most massive stars. The time spent on the Hayashi track depends on the adopted birthline. This results in a different transfer of angular momentum from the star to the disk, which in turn can change the angular momentum at the beginning of the MS phase.

In these fully or partly convective phases, the treatment of convection affects the location in the HR diagram, creating a significant difference in effective temperature. Figure 5 (cp) shows the location of the Hayashi tracks for models computed with the FST treatment of convection (dashed curves) and with the MLT treatment $(\alpha=1.6)$ (faded curves). The difference in effective temperature is of the order of $200 \mathrm{~K}$. As the convective envelope recedes, the tracks become undistinguishable.

However, the extremely thin surface convection zone which remains on the main sequence is affected (Figure $5(\mathrm{rp})$ ). The more superadiabatic the temperature gradient the less efficient is the convection. For a $1.8 M_{\odot}$, it can make the convective He II ionization zone appear or disappear. This is important since the thickness of the mixed superficial layers is crucial in explaining the abundance anomalies.

\subsection{Gravitational settling}

With such thin convective envelopes, gravitational settling is very efficient. Figure 6 illustrates this point.

In the left panel, a duck-star has all its chemical elements (chicken) showing at the surface. In the middle panel, the MS phase is symbolized by the crossing of a grid. If radiative forces are not taken into account together with gravitational settling, the duck-star ends its MS phase with only one element (chick) remaining at the surface.

However, radiative forces can in turn be too efficient and another mechanism must enter the game. It is the turbulent mixing which will not only affect the surface abundances but will induce changes in the internal structure as well, especially in the thickness of the convective envelope (Richard et al. 2001). One striking example is the formation of a convective iron zone at a temperature of $200000 \mathrm{~K}$ caused by an accumulation of ironpeak elements as a result of radiative forces. According to the mass of the star, this zone can merge with the H-He ionisation zone during the main sequence or remain detached, which affects the surface abundances of the iron-peak elements. 


\section{A-type stars: rotation}

From their location in the HR diagram, A-type stars are expected to rotate rapidly and not to be affected by magnetic braking. They can reach indeed rotational velocities up to $300 \mathrm{~km} \mathrm{~s}^{-1}$. Abt \& Morrel (1995, hereafter AM95) showed, however, that the distribution of rotational velocities has a bimodal shape, with virtually all the Am and Ap stars having equatorial rotational velocities $\left(v_{\text {rot }}\right)$ less than $120 \mathrm{~km} \mathrm{~s}^{-1}$, and most of the normal A0-F0 main-sequence stars having $v_{\text {rot }}>120 \mathrm{~km} \mathrm{~s}^{-1}$. They concluded (see also Abt (2000)) that rotation alone can explain the occurrence of abnormal or normal main-sequence A stars, and that the apparent overlap between their $v_{\text {rot }}$ distributions is only due to our inability to distinguish marginal Am stars from normal ones, or to disentangle rotational and evolutionary effects.

Considerable observational data indicates that during the MS evolution of A-type stars: 1) mass loss is limited to $\sim 2.10^{-10} M_{\odot} \mathrm{yr}^{-1}$. (Lanz \& Catala 1992); 2) rotational velocity does not depend on age; and 3) no significant angular momentum loss by magnetic braking is observed (e.g. Wolff \& Simon 1997, Hubrig et al. 2000). Consequently, the observed $v_{\text {rot }}$ distribution must be determined by the angular momentum evolution during the pre-main sequence (PMS) phase. In a study of the distribution of angular momentum in Orion stars, Wolff et al. (2004) find this hypothesis consistent with a simplified model of PMS evolution in which angular momentum is lost by an interaction with the protostellar disk during the convective phase, and conserved in the radiative one. Furthermore a core-envelope decoupling occurs during the convective-radiative transition (see also Stȩpień (2000) and Stȩpień \& Landstreet (2002) for Ap PMS angular momentum evolution). The understanding of angular momentum evolution during the PMS and the MS is fundamental to understand the Ap and the Am phenomena.

\subsection{Modelling the evolution of a rotating star}

Rotation has several different effects on stellar evolution: change of the internal hydrostatic equilibrium, changes in the apparent effective temperature and luminosity (Pérez Hernández et al. 1999 and references therein), transport of chemicals and of angular momentum by shears in differentially rotating stars, by meridional circulation and by horizontal turbulence (e.g., Zahn 1974, Knobloch \& Spruit 1982).

The effect of rotation on stellar evolution has been treated with different approaches: Endal \& Sofia (1981) assigned to each transport process (including meridional circulation) a diffusion coefficient, while Zahn (1992a) and Maeder \& Zahn (1998) hypothesized that differential rotation in the radiative zone of a nonmagnetic star gives rise to anisotropic turbulence (much stronger in the horizontal direction than in the vertical one due to the stratification) and results in "shellular" rotation. In this model the effective diffusion coefficient for the chemicals is:

$$
D_{\text {eff }}=\frac{|r U(r)|^{2}}{30 D_{\mathrm{h}}}, \text { with } D_{\mathrm{h}}=\frac{r}{C_{\mathrm{h}}}\left|\frac{1}{3 \rho r} \frac{\mathrm{d}\left(\rho r^{2} U\right)}{\mathrm{d} r}-\frac{U}{2} \frac{\mathrm{d} \ln r^{2} \Omega}{\mathrm{d} \ln r}\right| .
$$

Where $U$ is the meridional circulation velocity, $C_{\mathrm{h}}$ is a free parameter related to the turbulent horizontal viscosity, and the other quantities have the habitual meaning. Palacios et al. (2003) have applied Zahn's modeling of rotation with the formulation by Maeder \& Zahn (1998) (including also microscopic diffusion but not radiative accelerations) to masses from 1.35 to $2.2 \mathrm{M}_{\odot}$. We recall two results of this application: 1 ) the rotation profile inside the star shows differential rotation mainly close to the convective core. 2) The models (computed without overshooting), show a wider MS when rotation is included. However, while the lower mass models increase their MS lifetime by a 20\%, the largest 
masses increase it by only $10 \%$ (see their Fig. 1). This trend is opposite to that observed in open clusters and in binary systems, where the fitting of observations requires to increase the overshooting parameter with the mass (i.e. Andersen et al. 1990, Ribas et al. 2000).

Recently, Maeder (2003) and independently Richard \& Zahn (1999) have updated the value for the horizontal diffusion coefficient, $C_{\mathrm{h}}$ by a factor of $10^{2}$ with respect to the value used in Maeder \& Zahn (1998). Its implementation in a stellar evolution code (Mathis et al. 2004) leads to: 1) enhanced mixing, and 2) significant changes in the profile of chemical mixing with depth (see their Fig. 2). To what degree will the new $D_{\text {eff }}$ (with a non-zero value until the boundary of the convective core) affect the MS width and the surface chemical abundances?

The scheme proposed by AM95 matches qualitatively well the predictions of the microscopic diffusion models (e.g., Richer et al. 2000), in the hypothesis that the extra-mixing required to decrease the microscopic diffusion is induced by rotation. Some problems are, however, left, for instance: 1) the "normal" late B and early A-type main-sequence stars show a true bimodal $v_{\text {rot }}$ distribution (Royer et al. 2005) ; 2) the binary V392 Carinae has a $v \sin i$ of $27 \mathrm{~km} \mathrm{~s}^{-1}$ and no peculiar abundances (Debernardi \& North 2001). 3) There is no correlation between the strength of chemical peculiarities and $v_{\text {rot }}$ (Erspamer \& North 2003). Models including microscopic diffusion and radiative accelerations show that the chemical abundances are very sensitive to the thickness of the mixed layer (e.g., Alecian 1996, Hui-Bon-Hoa, 2000, Richard et al. 2001), so that we should expect a signature of $v_{\text {rot }}$ in the abundances, if rotation is the responsible of the extra mixing below the convective envelope. A first approach to explicitly include a model of rotationally induced mixing and a complete treatment of microscopic diffusion is in progress (Richard et al. 2005) .

Finally, there are also other effects that should be taken into account in the study of the evolution of a rotating A-type star:

1. Interaction convection-rotation. Rotation can reduce the efficiency of convection and is able to reduce the extension of the overshooting region (e.g., Julien et al. 1997). Recent numerical simulations of a rotating convective core (Browning et al. 2004) show that rotation leads to a variation of convective overshooting and penetration, and induces internal waves, meridional circulation, and differential rotation at the core boundary.

2. Magnetic field. Maeder \& Meynet (2003) have shown that the Tayler-Spruit magnetic instability (Spruit 2002) could take place in the interior of stars with small magnetic fields and differential rotation, induce a process of angular momentum transport much more efficient than that due to the meridional circulation and horizontal turbulence, and lead to solid body rotation on a short timescale. Furthermore, in Ap stars, which have undergone strong braking during their PMS phases (Stȩpień 2000), differential rotation together with a magnetic field could lead to magnetorotational instabilities. These could transport angular momentum from the interior to the surface (Arlt et al. 2003, and Artl 2005), and result in solid body rotation of these stars, as expected from some observational data (Hubrig et al. 2000).

\section{A-type stars: binarity}

Binarity plays a fundamental role in the origin and definition of the chemical peculiarities of A-type stars. The pioneering work of Abt $(1961,1965)$ showed that most (he even suggested all) Am type stars are relatively short period binaries and that the period distribution of binaries with Am and with non-peculiar A-type components are complementary. Normal A-type components are found in systems with period shorter 
than $\sim 2.5$ or longer than $\sim 100$ days, while Am stars form binaries with period in the range $2.5-100$ days.

Abt's statements have somewhat lost their strength with time and with the increasing size of studied binary samples. Some overlap between the period distribution of normal and Am binaries was found by the same author (AM95) and the binary frequency among Am stars has steadily decreased with time (see Abt \& Levy (1985) and North et al. 1998, Debernardi (2000) for results based on CORAVEL surveys). The latter author finds a frequency as low as $57 \%$ (but CORAVEL samples are certainly biased against fast rotation and, because of the limited time span of the survey, against longer period-eccentric orbit binaries). The - at any rate - high frequency has a straightforward explanation: in close binaries the spin-orbit synchronization by tidal mechanisms can efficiently brake the stellar rotation to values compatible with the Am phenomenon.

The other relevant connection between binarity and A-type star peculiarity is in the low frequency of spectroscopic binaries among the magnetic Ap stars (Abt \& Snowden 1973, Gerbaldi et al. 1985, North et al. 1998). The current explanation is that the strong magnetic fields prevent close binary formation (however, Budaj (1999) suggested instead that it is binarity which affects magnetism and not the other way around).

While the general outline of the binarity-peculiarity connection is well established, there are still shortcomings in the theory of tidal synchronization. Besides, possible drawbacks in the interpretation of the observations are caused by the origin and composition of the observed samples (biases, selection effects).

Two competing theories were developed to explain the observed levels of orbital circularization and spin-orbit synchronization in close binaries: the tidal theory of Zahn (1992b) and references therein and the hydrodynamical theory of Tassoul \& Tassoul (1992) and references therein. The necessary ingredients of the first are tidal bulges and an efficient dissipation mechanisms; in absence of synchronism, dissipation causes a lag of the bulge and hence a torque, which tends to establish synchronization (and orbit circularization). The dissipation mechanisms at work are different for late and early type stars, in the first case it is turbulent dissipation in the convective envelope retarding the equilibrium tide, in the second it is radiative damping acting on the dynamical tide (forced gravity waves are emitted from the lagging convective core and are damped in the outer layers).

Tassoul's theory is based, instead, on the idea that while the torque due to dissipation processes is negligible, transient strong meridional currents are produced by the tidal action and transfer angular momentum between the stellar interior and an "Ekman layer" close to the surface. As a consequence if the rotation period is shorter than the orbital one, the star is braked.

Both theories yield time-scales for synchronization $\left(t_{m s}\right)$ and circularization $\left(t_{m c}\right)$. For early type stars, in Zahn's case:

$$
\frac{1}{t_{m s}} \propto\left(\frac{G M}{R^{3}}\right)^{\frac{1}{2}} q^{2}(1+q)^{\frac{5}{6}} \frac{M R^{2}}{I} E_{2}\left(\frac{R}{a}\right)^{\frac{17}{2}} ; \quad \frac{1}{t_{m c}} \propto\left(\frac{G M}{R^{3}}\right)^{\frac{1}{2}} q(1+q)^{\frac{11}{6}} E_{2}\left(\frac{R}{a}\right)^{\frac{21}{2}}
$$

where $q$ and $a$ are the mass ratio and the semi-axis of the binary, $M, R, I$ the mass, the radius and the inertial moment of the braked star and $E_{2}$ a constant related to the size of its convective core. In Tassoul's theory:

$$
\frac{1}{t_{m s}} \propto 10^{\frac{N}{4}-\gamma} q(1+q)^{\frac{3}{8}}\left(\frac{M L^{2}}{R^{9}}\right)^{\frac{1}{8}}\left(\frac{R}{a}\right)^{\frac{33}{8}} ; \frac{1}{t_{m c}} \propto 10^{\frac{N}{4}-\gamma}(1+q)^{\frac{11}{8}} \beta^{2}\left(\frac{M L^{2}}{R^{9}}\right)^{\frac{1}{8}}\left(\frac{R}{a}\right)^{\frac{49}{8}}
$$



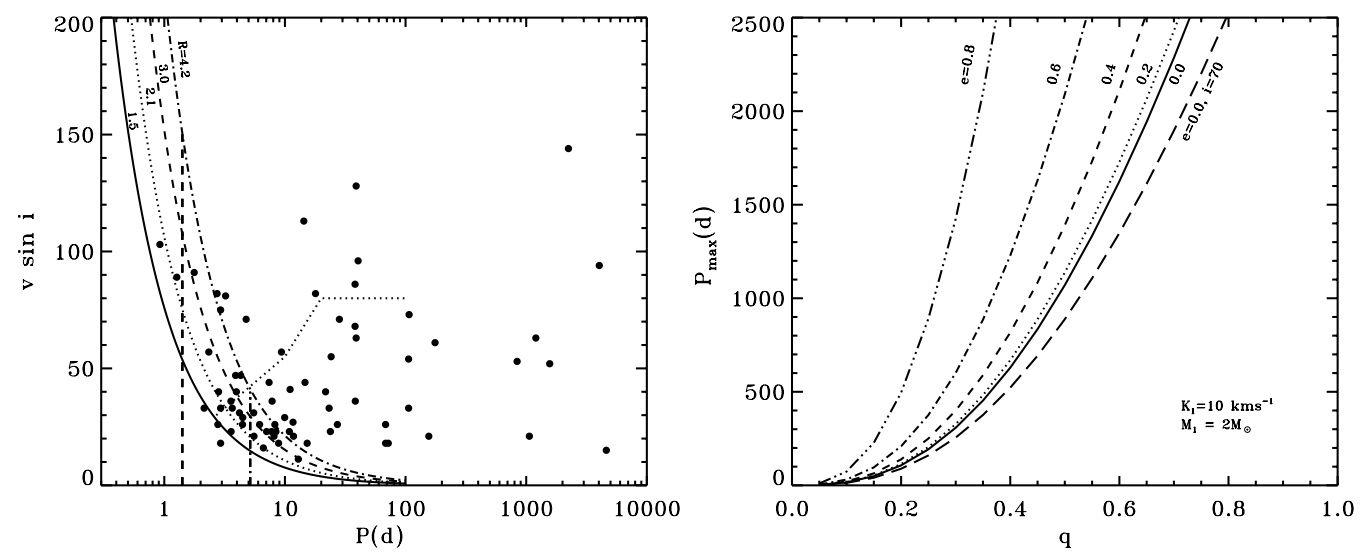

Figure 7. Left panel: The rotational velocities $(v \sin i)$ of spectroscopic binaries with Am components versus orbital period. The hyperbolic curves give the theoretical relation $\left(i=90^{\circ}\right)$ for synchronized systems of $2 M_{\odot}$ and different radii. The discontinuous dotted line is the lower boundary of the "avoidance zone", according to Budaj (1996), the vertical lines are the expected upper boundary for circularization, according to North \& Zahn (2003): dotted, $R=2.1 R_{\odot}$, $q=1$; dash-dotted, $R=3 R_{\odot}, q=0.2$. Right panel: The longest orbital period of a SB1 binary, corresponding to the assumed minimum observable radial velocity amplitude $K_{1}=10 \mathrm{~km} \mathrm{~s}^{-1}$, is plotted as function of mass ratio and eccentricity for primary mass $M=2 M_{\odot}$ and inclination $i=90^{\circ}$

with $L$ being the star luminosity, $\beta$ its fractional gyration radius, $N$ the ratio between eddy and radiative viscosity ( $N=0$ for radiative envelopes). The other factor, $\gamma$, was introduced by Claret et al. 1995) to take somehow into account the fact that the orbital evolution is derived (as in Zahn's case) under the hypothesis of small deviations from synchronism and from circular orbit. The circularization time is in general two/three orders of magnitude longer than that of synchronization, due to the larger amount of angular momentum stored in the orbit. It is evident by comparing Eqs. 4.1 with 4.2 that Tassoul's mechanism has a longer range and a much higher efficiency, especially for early-type stars. The timescales are - at any rate - only an indication of the process speed, and cannot replace the full integration of the orbital evolution equations.

In principle the comparison between the expected and the observed degree of synchronization/circularization in binaries of known age and accurate dimensions could discriminate between the two theories, and early type stars are the best choice for such a test. However, the detailed treatment of Claret et al. 1995) and Claret \& Cunha (1997) (with simultaneous computation of orbital and stellar evolution and a comparison sample of observed binaries with the best known parameters) showed that the results are still inconclusive, as both models can explain only a part of the observations. Zahn's mechanism is not efficient enough for early type stars (but the pre-MS phases were not considered in the abovementioned papers) while Tassoul's one is too efficient, unless a high value of $\gamma$ is introduced (to shorten the time-scales by a factor $\sim 40$ ). The most promising scenario remains, therefore, that suggested in a study of circularization in binaries with A-type components by Matthews \& Mathieu (1992): a process imposed by an important PMS phase of orbital evolution followed by a MS phase, which however still awaits full modeling.

The recent survey of A-type star rotational velocities by Royer et al. (2002), hereafter R02, provides excellent material for the study of synchronization in binaries with Am components. Figure 7 shows the updated $v \sin i$ values from R02 versus orbital period, for the Am binaries listed in Budaj (1996), and the relations expected in case of spin-orbit 
synchronization. The vertical lines are the periods corresponding to the fractional radius $(R / a)$ that should assure circularization (North \& Zahn (2003)) - and presumably circularization - the case of a $2 M_{\odot}$ MS-primary. The data are generally in good agreement with the expectations, with a marginal indication of radii larger than in normal stars (as the synchronization relations should be upper envelopes, having assumed $i=90^{\circ}$ ).

The R02 velocities are generally higher than those measured by AM95 and used by Budaj; the reason is a systematic effect in the velocity of standard stars used by AM95. As a consequence the "avoidance zone", found by Budaj (1996), for periods $4<P<20$ days (i.e., the region above the dotted line in Fig. 7) is no longer empty and it is not necessary for an ad-hoc mechanisms to explain it, such as the "tidal mixing" introduced by the abovementioned author; the decreasing number of systems found in proximity of the synchronism lines can be due to a dependence on period of the braking efficiency.

The other feature appearing in Figure 7, the lack of systems between $180<P<800$ days, is quite probably a selection effect due to the low probability of discovery as a spectroscopic binary. An idea of the relevance of selection effects can be derived from the right panel of Fig. 7 . This shows, for a $M=2 M_{\odot}$ primary, the largest orbital period corresponding to a given minimum observable radial velocity amplitude, $K_{1}$. This is a function of mass ratio, $q$, and eccentricity, $e$ (in the hypothesis of inclination $i=90^{\circ}$ ). Even with the conservative hypothesis $K_{1}=10 \mathrm{~km} \mathrm{~s}^{-1}$ (but Hogeveen (1992), on the basis of a detailed study of selection effects for spectroscopic binaries, suggests $25 \mathrm{~km} \mathrm{~s}^{-1}$ for A-type stars) Figure 7 indicates that the largest period of binaries detectable by spectroscopic surveys is of some hundred days (and many systems will escape detection, as the distribution of mass ratio of single lined spectroscopic binaries is peaked around $q=0.2$ ). The gap in the period distribution could, therefore, be due to the transition between spectroscopic binaries, discovered as such, and visual binaries with known radial velocity.

In conclusion, a proper treatment of selection effects is a primary issue to solve if we want to extract correctly the rich information that A-type stars can provide on binary secular evolution and that, in their turn, binaries can yield on A-type star properties.

\section{Acknowledgements}

The authors thank the IAU for grants covering the registration fees. A.N and J.M. acknowledge ESA-PRODEX contract 15448/01/NL/SFe(IC)-C90135 and IAP P5/36, and C.M. a F-INAF program for funding.

\section{References}

Abt, H.A. 1961, ApJS 6, 37

Abt, H.A. 1965, ApJS 11, 429

Abt, H.A. 2000, ApJ, 544, 933

Abt, H.A. \& Levy, S.G. 1985, ApJS 59, 229

Abt, H.A. \& Morrell, N.I. 1995, ApJS 99, 135 (AM95)

Abt, H.A. \& Snowden, M.S. 1973, ApJS 25, 137

Andersen, J., Nordström, B., \& Clausen, J.V. 1990, ApJ 363, L33

Arlt. R. 2005, These Proceedings, 103

Arlt, R., Hollerbach, R., \& Rüdiger, G. 2003, A\& A 401, 1087

Behrend, R. \& Maeder, A. 2001, A\& $A$ 373, 190

Browning, M.K., Brun, A.S., \& Toomre, J. 2004, ApJ 601, 512

Budaj, J. 1996, A\&SA 313, 523

Budaj, J. 1999, MNRAS 310, 419 
Chan, K.L. 1996, in Cool Stars, Stellar Systems, and the Sun, 9th Cambridge Workshop, ASP Conf. Ser. 109, p. 561

Claret, A. \& Cunha, N.C.S. 1997, A\&\&A 318, 187

Claret, A., Gimenez, A., \& Cunha, N.C.S. 1995, A\&A 299, 724

Debernardi, Y. 2000, IAU Symposium 200, p. 161

Debernardi, Y. \& North, P. 2001, A\&A 374, 204

Endal, A.S. \& Sofia, S. 1981, ApJ 243, 625

Erspamer, D., \& Norht, P. 2003, A\&A 398, 1121

Formicola, A. \& LUNA 2004, Physical Letters $B$ 591, 61

Gerbaldi, M., Floquet, M., \& Hauck, B. 1985, A\&A A, 146, 341

Hogeveen, S.J. 1992, ApESSS 196, 299

Hubrig, S., North, P., \& Medici, A. 2000, A\&A, 359, 306

Hui-Bon-Hoa, A. 2000, A\&ASS 144, 203

Julien, K., Werne, J., Legg, S., \& McWilliams, J. 1997, in SCORe'96 : Solar Convection and Oscillations and their Relationship, ASSL Vol. 225, p. 227

Knobloch, E. \& Spruit, H.C. 1982, A\&̈A 113, 261

Lanz, T. \& Catala, C. 1992, A\&A, 257, 663

Mathis, S., \& Zahn J.-P. 2004, A\&A in press

Mathis, S., Palacios, A., \& Zahn J.-P. 2004, $A \& A$ in press

Maeder, A. 2003, AESA 399, 263

Maeder, A., \& Meunet, G. 2003, A\&SA 411, 543

Maeder, Z., \& Zahn, J.-P. 1998, A\&SA 334, 1000

Matthews, L.D. \& Mathieu, R.D. 1992, in IAU Colloq. 135: Complementary Approaches to Double and Multiple Star Research, ASP Conf. Ser. 32, p. 244

North, P., Ginestet, N., Carquillat, J.-M., Carrier, F., \& Udry, S. 1998, Contributions of the Astronomical Observatory Skalnate Pleso 27, 179

North, P. \& Zahn, J.-P. 2003, A\&A 405, 677

Palacios, A., Talon, S., Charbonnel, C., \& Forestini, M. A\& $A$ 399, 603

Palla, F. \& Stahler, S. W. 1993, ApJ 418, 414

Pérez Hernández, F., Claret, A., Hernández, M.M., \& Michel, E. 1999, A\&A A 346, 586

Ribas, I., Jordi, C., Tora, Torra, J., Giménez, Á. 2000, MNRAS, 313, 99

Richard, D. \& Zahn, J.-P. 1999, A\&A 347, 734

Richard, O., Michaud, G., \& Richer, J. 2001, ApJ 558, 377

Richard, O., Talon, S., Michaud, G., 2005, These Proceedings, 215

Royer, F., Grenier, S., Baylac, M.-O., Gómez, A.E., \& Zorec, J. 2002, A\&3A 393, 897 (R02)

Royer, F., Zorec, J., Goméz, A. E., 2005, These Proceedings, 109

Spruit, H.C. 2002, A\&SA, 381, 923

Stȩpień, K. 2000, A\& $A$ 353, 227

Stȩpień, K. \& Landstreet, J.D. 2002, A\&A 385, 554

Tassoul, J. \& Tassoul, M. 1992, ApJ 395, 259

Wolff, S.C., \& Simon, T. 1997, PASP 109, 759

Wolff, S.C., Strom, S.E., \& Hillenbrand, L.A. 2004, ApJ 601, 979

Zahn, J.-P. 1974, in IAU Symp. 59: Stellar Instability and Evolution, p. 185

Zahn, J.-P. 1992a, A $\& A$ 265, 115

Zahn, J.-P. 1992b in A. Duquennoy \& M. Mayor (eds), Binaries as Tracers of Stellar Formation. Cambridge: Cambridge University Press, p. 253

\section{Discussion}

Discussion postponed to the panel discussion B (see pp. 115-118, These Proceedings). 